\title{
Kidney disease susceptibility may be drawback of parasite resistance in African Americans
}

T

wo independent variants of APOL1

that are associated with renal disease probably evolved from their ability to resist infectious disease, according to a new study. "We found that specific $A P O L 1$ variants with high frequency in African Americans increase the risk of kidney disease and protect against Trypanosoma brucei rhodesiense infection", explains the study's corresponding author, Martin Pollak.

African Americans are more susceptible than white Americans to certain forms of kidney disease, such as focal segmental glomerulosclerosis (FSGS) and hypertension-induced end-stage renal disease (ESRD). Previous studies identified an association between risk of kidney disease in these individuals and genetic variation at a locus near MYH9 on chromosome 22; however, causal mutations in MYH9 have not been identified and recent findings led Pollak and colleagues to expand their search. "In the course of our research, we did a genome-wide association scan of patients with FSGS, which made us suspect that the major gene accounting for the high rate of FSGS in black individuals could be located in a larger genomic region than originally suspected", says Pollak.

To investigate whether variations in this expanded chromosomal region may be responsible for the increased association of renal disease in black individuals, Pollak and colleagues analyzed sequence data from the 1,000 Genomes Project to identify polymorphisms that markedly differed in frequency between Africans and Europeans. They then compared sequence variations between 205 AfricanAmerican individuals with biopsyproven FSGS and 180 African-American individuals with no evidence of renal disease. The researchers identified two variations in the APOL1 gene-termed G1 and G2-that correlated with disease. "Comparing subjects with zero or one risk allele to subjects with two risk alleles

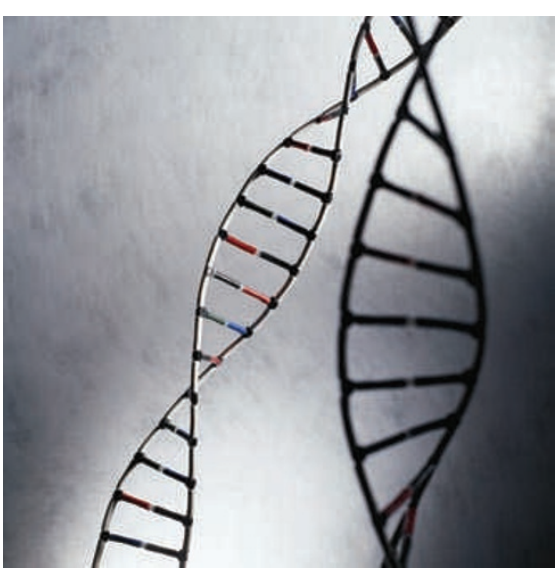

provided an odds ratio for FSGS of 10.5 ([95\%] CI 6.0-18.4)", say the researchers.

Pollak and colleagues also tested the association of $A P O L 1$ variants with hypertension-induced ESRD in a larger cohort of 1,030 African Americans with disease and 1,025 geographically matched African Americans without disease. The G1 and G2 variants were again found to be strongly associated with disease. "The statistical significance of the combined signal ... was 35 orders of magnitude stronger than for MYH9 SNPs [single nucleotide polymorphisms]".

An analysis of different populations revealed that the G1 and G2 variants were present in chromosomes from African individuals but not in chromosomes from European, Japanese, or Chinese individuals, leading Pollak and colleagues to speculate that these variants may confer a selective advantage to individuals from Africa. This hypothesis was tested in collaboration with Etienne Pays. Previous work by Pays and colleagues had demonstrated that ApoL1 could kill typical trypanosomes. "As soon as we discovered the papers mentioning the possible association between MYH9 and a disease typically frequent in people of recent African origin, we immediately speculated that the renal disease was actually linked to changes in expression of the APOL1 gene, which is located immediately upstream of MYH9", says Pays.

The researchers found that the APOL1 variants could kill trypanosomes that were normally resistant to ApoL1 toxicity. "We were struck by the fact that the two APOL1 mutants were able to kill the human pathogen T. brucei rhodesiense", states Pays. "To me, the most significant finding was that the two mutations that characterize the G1 phenotype were individually only weakly toxic to T. brucei rhodesiense but fully lytic under physiological conditions when present together, as happens in nature. This finding strongly suggests that G1 was selected to resist T. brucei rhodesiense".

Pollak and colleagues suggest that the variants' lytic activity provides a plausible biological explanation for the natural selection of these mutants in individuals of recent African descent, but further questions remain to be answered. "It is interesting that the $A P O L 1$ variants are particularly present in West Africa-a region where T. brucei rhodesiense cannot be found today. We would like to evaluate the possibility that the selection of these mutants prevented the spreading of this parasite, favoring instead the emergence of another African trypanosome, T. brucei gambiense, which is resistant to lysis by both G1 and G2", says Pays. In addition, Pollak acknowledges that their findings do not elucidate the mechanism by which these variants increase susceptibility to kidney disease. "We would like to sort out exactly how these variants lead to kidney disease, as improved understanding of the mechanisms involved may help classify patients and tailor treatments", concludes Pollak.

Susan J. Allison 